\title{
MICROSTRUCTURAL CHANGES OF CARBONACEOUS MONOLITHS SYNTHESIZED VIA HYDROTHERMAL METHOD
}

\author{
H.N. LIM", ${ }^{1, *}$ A. KASSIM², S.P. LIM², N.S. RASTAM NIZAR², N.M. HUANG \\ ${ }^{I}$ Centre for Ionics University of Malaya, Physics Departament, Faculty of Science, University of Malaya, 50603 Kuala Lumpur, Malaysia \\ ${ }^{2}$ Chemistry Department, Faculty of Science, Universiti Putra Malaysia, 43400 UPM Serdang, Selangor Darul Ehsan, Malaysia. \\ ${ }^{3}$ Low Dimensional Materials Research Centre, Phsics Departament, Faculty of Science, University of Malaya, 50603 Kuala Lumpur, Malaysia.
}

(Received: April 21, 2010 - Accepted: September 9, 2010)

\begin{abstract}
Carbonaceous monoliths were successfully synthesized via a facile hydrothermal processing route using phenol as a carbon precursor. The x-ray diffraction (XRD) patterns revealed distinguishable (002) and (100) planes of graphite at approximately $2 \theta=23^{\circ}$ and $44^{\circ}$, respectively. The fourier transform infrared (FTIR) spectroscopy corresponded to the chemical bonds of graphite, which were $\mathrm{C}=\mathrm{C}$ and $\mathrm{C}-\mathrm{H}$. The carbonaceous monoliths exhibited interesting morphological changes as a result of varying the type of polymer which acted as a structure directing agent, mass of polymer, mass of phenol and hydrothermal temperature before and after calcination.
\end{abstract}

Keywords: Carbon, graphite, hydrothermal, scanning electron microscope

\section{INTRODUCTION}

Carbon monoliths have attracted great interest in many fields, such as electrochemistry, energy storage, separation and chromatography, mainly due to their high thermal and chemical stabilities, large surface areas and substantial advantages concerning mass transport. ${ }^{1}$

Various approaches towards the synthesis of carbon monoliths have been conducted, including nanocasting, ${ }^{2}$ organic-organic self-assembly strategy via a solvent evaporation-induced self-assembly (EISA) method ${ }^{3}$ and common aqueous reaction routes under atmospheric pressure at relatively low temperatures. ${ }^{4}$ The drawbacks of 'nanocasting' are that this strategy is somewhat fussy and costly given its complicated multiple step synthetic procedures, and sacrificial use of both surfactants and monolithic silica templates. Similarly, the latter two methods are problematic for large-scale industrial production because of long processing times and the large vessels used for the interface assembly of mesostructures. ${ }^{1}$

Hydrothermal synthesis is a more efficient approach under controlled temperature and pressure that has been extensively exploited in a variety of inorganic syntheses of zeolites, nanomaterials, catalysts and ion-conductors. In addition, this method is faster and more energy efficient than conventional aqueous chemical processing conditions. ${ }^{1}$

Herein, we report observation of various morphologies of carbon monoliths synthesized using hydrothermal method. The processing route was varied by different parameters such as type of polymer which acted as a structure directing agent, mass of polymer, mass of phenol and hydrothermal temperature. Interesting morphological features were observed using a scanning electron microscope. The crystallinity and chemical bonding of the carbonaceous monoliths were investigated using an $\mathrm{x}$-ray diffractometer and a fourier transform infrared spectrometer, respectively.

\section{EXPERIMENTAL}

Carbonaceous monoliths were synthesized based on Huang et al. (2009). Briefly, $3.5 \mathrm{ml}$ of formaldehyde solution ( $37 \mathrm{wt} \%$, HmbG Chemical) and 0.1 g of industrial grade phenol (99\%) were dissolved in $5 \mathrm{ml}$ of $0.5 \mathrm{M}$ sodium hydroxide $\left(85 \%\right.$, Merck) solution and stirred at $70^{\circ} \mathrm{C}$ for $30 \mathrm{~min}$, in which the colorless solution turned yellow. Then, $2.0 \mathrm{~g}$ of PEG-1 000 was added to the above solution and the mixture was continuously stirred at $70^{\circ} \mathrm{C}$ for an additional $3 \mathrm{~h}$. After that, the pinkish slurry was poured into a Teflon-lined autoclave and transferred into an oven at $100^{\circ} \mathrm{C}$ for $10 \mathrm{~h}$. The brown polymeric monolith was washed with water and dried in air. The sample was calcined at $600^{\circ} \mathrm{C}$ for $3 \mathrm{~h}$ in a nitrogen flow to obtain black carbonaceous monolith. Table
1 shows the various parameters of synthesizing carbon monoliths.

Table 1. Synthesis parameters of carbon monoliths.

\begin{tabular}{|c|c|c|c|c|}
\hline Sample & $\begin{array}{l}\text { Type of } \\
\text { polymer }\end{array}$ & $\begin{array}{c}\text { Mass of } \\
\text { polymer }(\mathrm{g})\end{array}$ & $\begin{array}{c}\text { Mass of } \\
\text { phenol (g) }\end{array}$ & Reaction \\
\hline A & PEG - 1000 & 2.0 & 0.10 & 100 \\
\hline B & PEG - 20000 & 2.0 & 0.10 & 100 \\
\hline $\mathrm{C}$ & PEG - 35000 & 2.0 & 0.10 & 100 \\
\hline $\mathrm{D}$ & PEG - 20000 & 3.0 & 0.10 & 100 \\
\hline $\mathrm{E}$ & PEG - 20000 & 3.5 & 0.10 & 100 \\
\hline $\mathrm{F}$ & PEG - 20000 & 2.0 & 0.15 & 100 \\
\hline $\mathrm{G}$ & PEG - 20000 & 2.0 & 0.20 & 100 \\
\hline $\mathrm{H}$ & PEG - 20000 & 2.0 & 0.30 & 100 \\
\hline $\mathrm{J}$ & PEG - 20000 & 2.0 & 0.10 & 60 \\
\hline
\end{tabular}

The crystallinity of the samples was measured using a Phillips X-Ray Diffractometry (XRD). The as-prepared sample was placed on a glass slide. Measurements were taken from $10^{\circ}$ to $50^{\circ}$ on the $2 \theta$ scale at a size step of $0.033^{\circ} \mathrm{s}^{-1}$. The XRD data was processed using in-built PANalytical X'pert HighScore software to examine the peak position and its corresponding intensity data. Chemical bonding of the samples was analyzed using a PerkinElmer Fourier Transform Infrared (FTIR) spectroscopy. Each sample was mixed with potassium bromate, ground homogenously and converted into pellets. The spectra (\% transmittance with wavenumber) were recorded. The morphology of the samples was observed using a LEO 1455 Variable Pressure Scanning Electron Microscopy (VPSEM). The samples were mounted on aluminium stubs using double-sided tape and vacuum coated with gold in a Polaron SC500 sputter coater.

\section{RESULTS AND DISCUSSION}

Figure 1 shows the XRD patterns of sample B before and after calcination, which represents all the other samples. The recorded two peaks at $2 \theta=23^{\circ}$ and $44^{\circ}$ are reflections from the (002) and (100) planes, respectively. The peaks are well-assigned to the structure of carbon with hexagonal phase (JCPDS 75$1621),{ }^{5}$ which is distinguishable to that of graphite. The (100) reflection at $44^{\circ}$ suggests honeycomb structures formed by $\mathrm{sp}^{2}$ hybridized carbons, while the (002) reflection at $23^{\circ}$ indicates small domains of coherent and parallel stacking of the graphene sheets. ${ }^{6}$ 


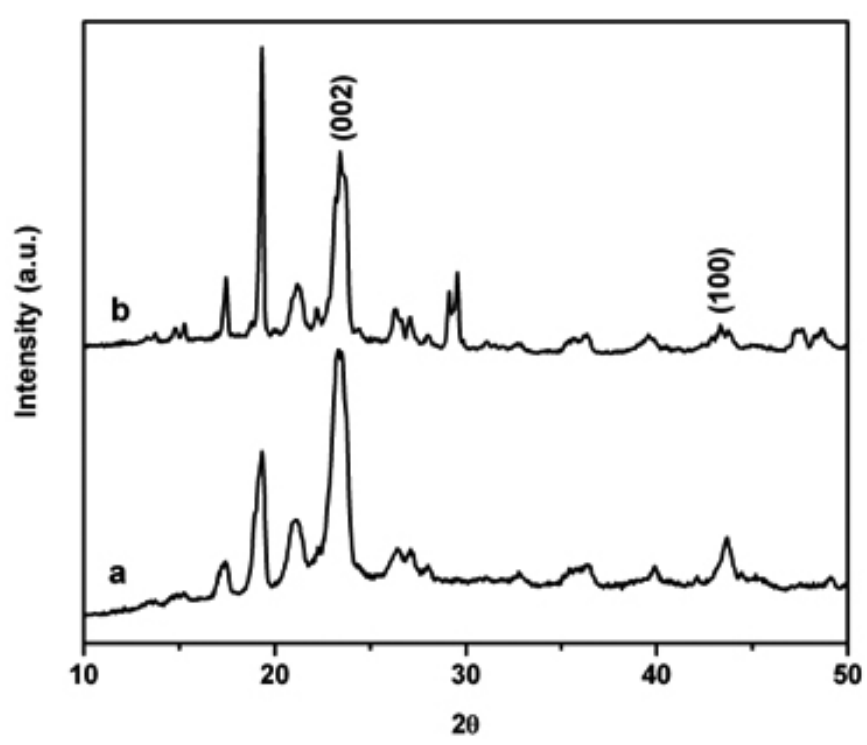

Figure 1. XRD patterns of sample B before (a) and after (b) calcination.

Figure 2 shows the FTIR spectra of sample B before and after calcination, representing the rest of the samples. Both the samples before and after calcination exhibit bands at $1520-1770 \mathrm{~cm}^{-1}$ attributed to $\mathrm{C}=\mathrm{C}$ vibrations and $750-875 \mathrm{~cm}^{-1}$ assigned to aromatic C-H out-of-plane bending vibrations. ${ }^{7}{ }^{8} \mathrm{In}$ contrast, only the samples before calcination exhibit bands at $1000-1450 \mathrm{~cm}^{-}$ ', $2922 \mathrm{~cm}^{-1}$ and $3000-3700 \mathrm{~cm}^{-1}$, corresponding to C-O stretching and $\mathrm{O}-\mathrm{H}$ bending, ${ }^{8,9}$ stretching vibrations of aliphatic $\mathrm{C}-\mathrm{H}$ and $\mathrm{O}-\mathrm{H},{ }^{10}$ respectively. The results indicate that a large amount of residues, including hydroxyl and carboxyl groups on the surface of the samples have been eliminated from calcination, leaving behind carbonaceous materials only, which complement the XRD results.

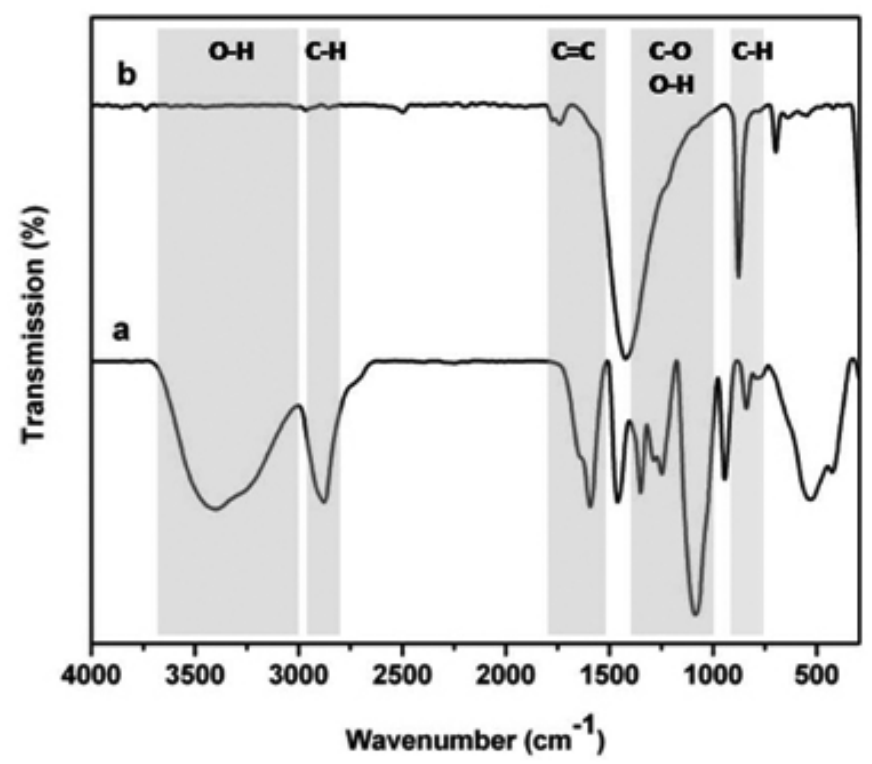

Figure 2. FTIR spectra of sample B before (a) and after (b) calcination.

Figures $3-6$ depict the morphological structures of monoliths before and after calcination. Generally, there are discernible feature changes in the monoliths before and after the calcination process contributed by the type of polymer which acted as a structure directing agent, mass of polymer, mass of phenol and hydrothermal temperature.
Polyethylene glycols (PEGs) with various molecular weights employed as templates were observed to influence the eventual morphologies of the monoliths before and after calcination (Figure 3). The lower the molecular weight of PEG such as PEG-1 000, the more bulbous the polymeric monolith (Figure 3a). The size of the bulbs decreased with increasing molecular weight of PEG- 20000 (Figure 3b). Further increment to PEG-35 000 shows a morphology that is free from the distinctive bulbs (Figure 3c). It is interesting to note the bulbs reappeared after calcination, including that of PEG-35 000 (Figures $3 \mathrm{~d}-3 \mathrm{f}$ ). The sizes of the bulbs are $5-7 \mu \mathrm{m}$ and $3-5 \mu \mathrm{m}$ before and after calcination, respectively. Moreover, there is hay-like morphology which appears alongside the bulbs. The hay lengthened but decreased in amount with increasing molecular weight of PEG, which could be due to the suppression by the large monomers. Based on our observation, the bulbs and hay were carbonaceous monoliths that are free from residues after calcination. The distinctive feature remains after removal of organic matters from calcination due to the template effect of PEGs. The larger the molecular weight of PEG, the ability of the monomers to envelope the carbon source will be heightened, as indicated by the absence of bulbs when PEG-35 000 was used as a template. In contrast, the small monomers, especially PEG-1 000, may lack the ability to converge the phenol, thus gives rise to the bulbous appearance.
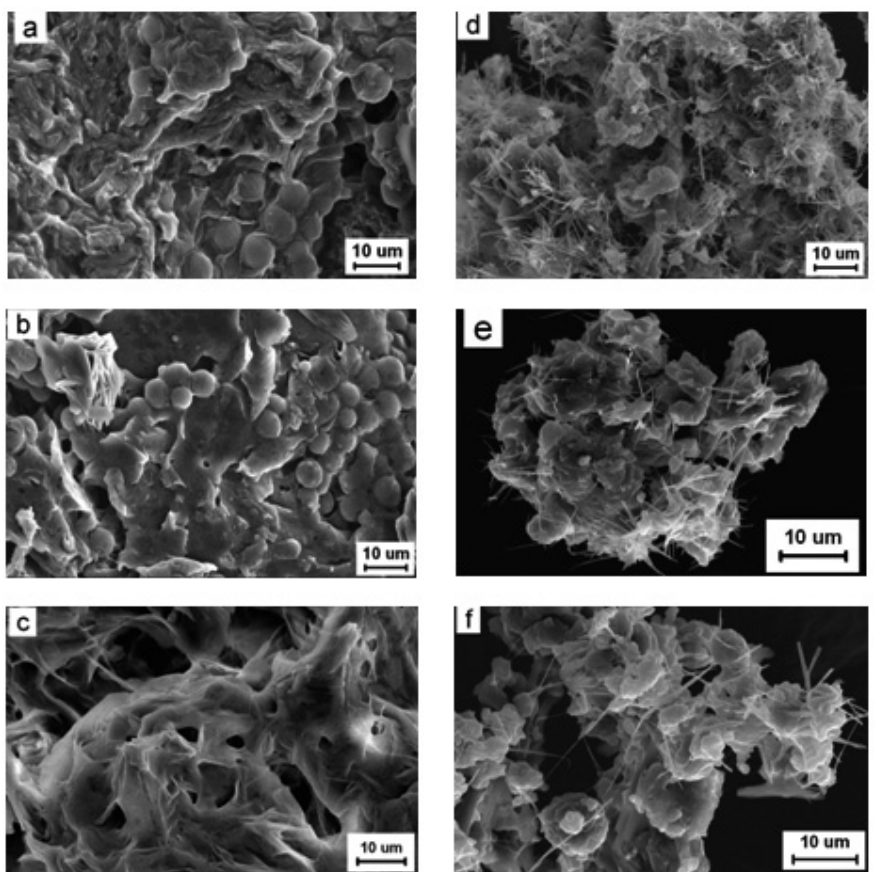

Figure 3. Scanning electron micrographs of samples $\mathrm{A}-\mathrm{C}$ before $(\mathrm{a}-\mathrm{c})$ and after $(\mathrm{d}-\mathrm{f})$ calcination.

The mass of PEG is also influential in determining the morphology of the monoliths. The polymeric monoliths before calcination portray increasing smoothness of the monoliths as expected (Figure 4). The higher mass of PEG (Figures $4 \mathrm{a}$ and $4 \mathrm{~b}$ ) is able to provide better coverage of the carbon precursor due to mechanical rigidity ${ }^{11}$ as oppose to the lower mass of PEG (Figures $3 b)$. This leads to purely hay-like morphology with length-to-width ratio of approximately 10 after calcination when appropriate amount of PEG-20 000 was used as a template (Figure 4c). The hay-like morphology was absent when $3.5 \mathrm{~g}$ of PEG-20 000 was used (Figure 4d). This is explainable by the fact that at such a large amount of PEG, the monomers overload the geometry of the template which gives rise to the disdented and stunted morphology of the particles in the carbonaceous monolith after the removal of template. 

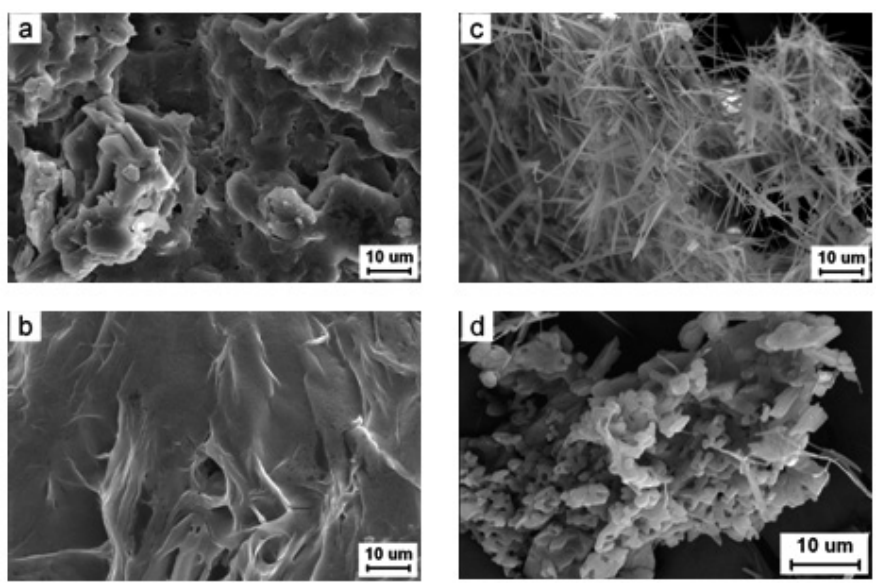

Figure 4. Scanning electron micrographs of samples D and $\mathrm{E}$ before (a and b) and after (c and d) calcination.

The mass of phenol plays an equally important role in the morphology of monoliths. The density of the pores decreased dramatically with increasing mass of phenol before and after calcination. The bulbous characteristic of the monolith (Figure $3 \mathrm{~b}$ ) changed to a network structure of monoliths before calcination (Figures $5 \mathrm{a}-5 \mathrm{c}$ ). A plausible explanation is that the amount of phenol becomes too overbearing for the monomers to constraint within the geometry ${ }^{12}$ and therefore, the formation of bulbs is restraint. The amount of the phenol overwhelms the porosity, which explains the minimum degree of porosity. After calcination, the carbonaceous monoliths exhibit particles which interconnected with one another, forming network structures (Figure $5 \mathrm{~d}$ and 5e). However, at $3.5 \mathrm{~g}$ of phenol, independent multi-faceted rods with an average ratio of length-to-width of 10 are observed (Fig. 5f). This may be explained by the fact that at such a large amount of phenol, the template was unable to maintain the formation of a network structure, causing the interconnections to collapse.
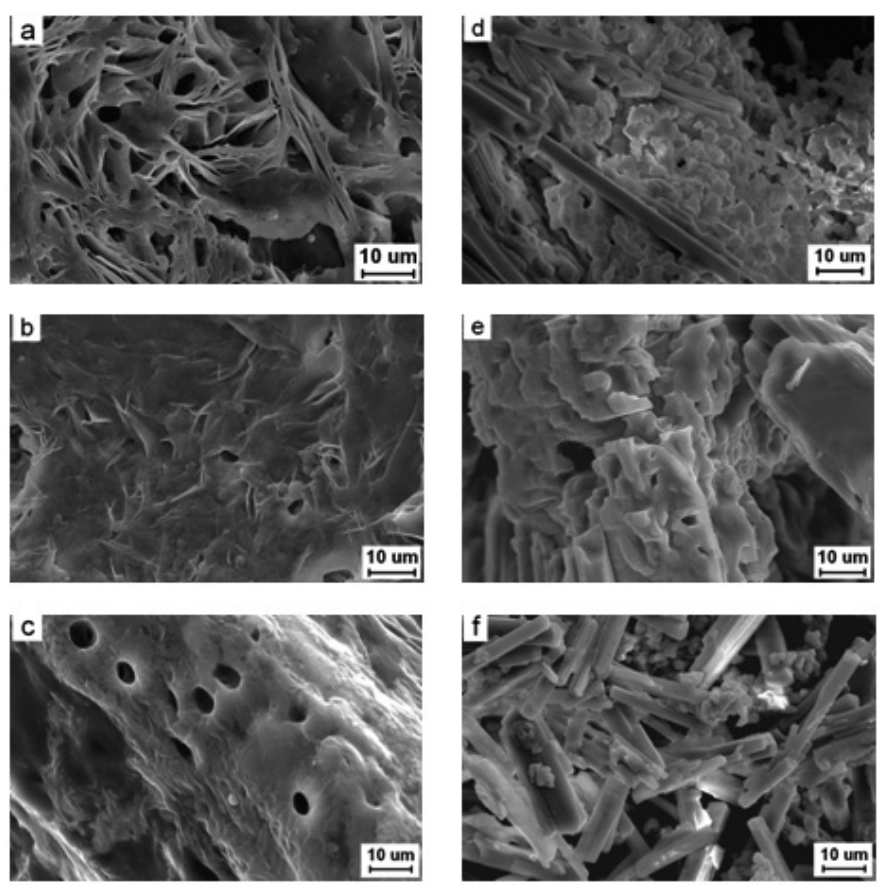

Figure 5. Scanning electron micrographs of samples $\mathrm{F}-\mathrm{H}$ before $(\mathrm{a}-\mathrm{c})$ and after $(\mathrm{d}-\mathrm{f})$ calcination.
The hydrothermal temperature affects the morphology of the monoliths before and after calcination. At a lower reaction temperature, the polymeric monolith appears to be wave-like. Upon scrutiny, the monolith is madeup of layers of sheets (Figure 6a), much unlike the bulbous appearance at a higher hydrothermal temperature. This could be because at a lower reaction temperature, the monomers of PEG and phenol just mixed with each other without going through a thorough templating process. This observation could also explain the indistinctive feature of carbonaceous monolith after calcination, which shows particles of about $3 \mu \mathrm{m}$ aggregating uncharacteristically (Figure $6 b)$.
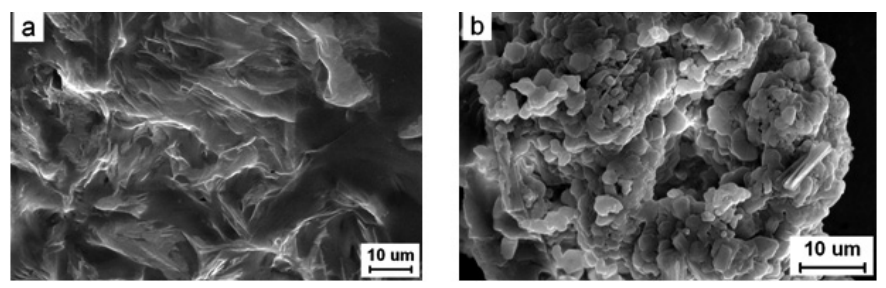

Figure 6. Scanning electron micrographs of sample J before (a) and after calcination (b).

\section{CONCLUSIONS}

We observed interesting three-dimensional carbonaceous monoliths synthesized using a facile hydrothermal method, in which their morphologies could be tailored according to interests by tuning different parameters. These monoliths could be widely employed in many applications such as catalyst supports in fuel cells, energy storage devices like batteries, electrochemical capacitors, absorbents and filter materials. The variety of morphologies of carbonaceous monoliths may intrigue many a scientist to probe further into correlating their morphologies to specific applications, which could manifest their potential applicability in many industrial sectors.

\section{REFERENCES}

1. Y. Huang, H. Cai, D. Feng, D. Gu, Y. Deng, B. Tu, H. Wang, P. A. Webley, D. Zhao, Chem. Comm. 2641, (2008).

2. Y. S. Hu, P. Adelhelm, B. M. Smarsly, S. Hore, M. Antonietti, J. Maier, Adv. Func. Mater. 17, 1873, (2007).

3. Y. Deng, T. Yu, Y. Wan, Y. Shi, Y. Meng, D. Gu, L. Zhang, Y. Huang, C. Liu, X. Wu, D. Y. Zhao, J. Am. Chem. Soc. 129, 1690, (2007).

4. F. Q. Zhang, Y. Meng, D. Gu, Y. Yan, C. Z. Yu, B. Tu, D. Y. Zhao, J. Am. Chem. Soc. 127, 13508, (2005).

5. M. Zheng, L. Liu, K. Jiang, Y. Xiao, D. Yuan, Carbon 48, 1224, (2010).

6. A. M. Stephan, T. P. Kumar, R. Ramesh, S. Thomas, S. K. Jeong, K. S. Nahm, Mater. Sci. Eng. A 430, 132, (2006).

7. A. C. Lua, T. Yang, J. Colloid Inter. Sci. 274, 594, (2004).

8. J. V. Ibarra, E. Munoz, R. Moliner, Org. Geochem. 24, 725, (1996).

9. T. Sakaki, M. Shibata, T. Mili, H. Hirosue, N. Hayashi, Bioresour. Technol. 58, 197, (1996).

10. M. SevillaA. B. Fuertes, Chem. Eur. J. 15, 4195, (2009).

11. H. N. Lim, A. Kassim, N. M. Huang, P. S. Khiew, W. S. Chiu, Colloids Surf. A 345, 211, (2009).

12. H. N. Lim, A. Kassim, N. M. Huang, R. Hashim, S. Radiman, P. S. Khiew, W. S. Chiu, Ceram. Inter. 35, 2891, (2009). 